\title{
A New Approach to Non-Fragile State Estimation for Continuous Neural Networks with Time-Delays
}

\author{
Fan Yang, Hongli Dong*, Zidong Wang, Weijian Ren and Fuad E. Alsaadi
}

\begin{abstract}
In this paper, the non-fragile state estimation problem is investigated for a class of continuous neural networks with time-delays and nonlinear perturbations. The estimator to be designed is of a simple linear structure without requiring the exact information of the activation functions or the time-delays, and is therefore easy to be implemented. Furthermore, the designed estimator gains are allowed to undergo multiplicative parameter variations within a given range and the non-fragility is guaranteed against possible implementation errors. The main purpose of the addressed problem is to design a non-fragile state estimator for the recurrent delayed neural networks such that the dynamics of the estimation error converges to the equilibrium asymptotically irrespective of the admissible parameter variations with the estimator gains. By employing a combination of the Lyapunov functionals and the matrix analysis techniques, sufficient conditions are established to ensure the existence of the desired estimators and the explicit characterization of such estimators are then given via solving a linear matrix inequality. Finally, a simulation example is used to illustrate the effectiveness of the proposed design method.
\end{abstract}

\section{Index Terms}

Recurrent neural networks; State estimation; Non-fragility; Time-delays; Lyapunov functional; Matrix inequality.

\section{INTRODUCTION}

For several decades, recurrent neural networks (RNNs) have been a focus of research mainly because of their capability of learning and approximating nonlinear functions in an adaptive way. So far, a variety of RNN architectures as well as RNN learning schemes have been used for pattern recognition, classification, regression and optimization problems with practical applications in many areas such as system identification and control, trajectory prediction, decision making and medical diagnosis [9], [13]. On the other hand, due to finite switching speed of amplifiers in electronic neural networks and finite signal

This work was supported in part by the National Natural Science Foundation of China under Grants 61422301 and 61374127, the Outstanding Youth Science Foundation of Heilongjiang Province under Grant JC2015016, the Technology Foundation for Selected Overseas Chinese Scholar from the Ministry of Personnel of China, and the Alexander von Humboldt Foundation of Germany.

F. Yang, H. Dong and W. Ren are with the College of Electrical and Information Engineering, Northeast Petroleum University, Daqing 163318, China. (Email: shiningdhl@vip.126.com)

Z. Wang is with the Department of Computer Science, Brunel University London, Uxbridge, Middlesex, UB8 3PH, United Kingdom. He is also with the Faculty of Engineering, King Abdulaziz University, Jeddah 21589, Saudi Arabia. (Email: Zidong.Wang@brunel.ac.uk)

F. E. Alsaadi is with the Faculty of Engineering, King Abdulaziz University, Jeddah 21589, Saudi Arabia.

* Corresponding author. 
propagation time in biological networks, the time-delays in signal transmission are often unavoidable. If not properly taken into account, the time-delays would cause the undesirable oscillation and even the instability. In the past 10 years or so, the time-delay phenomenon has received considerable research attention in the dynamic analysis problems for various neural networks [6], [8], [12], [17], [33]. For example, in [35], a kind of observer-based adaptive neural network controller has been designed for a class of single-input single-output strict-feedback nonlinear stochastic systems with unknown time-delays. In [26], the problem of global exponential stability of static neural networks with time delay and impulses has been investigated by using the Lyapunov functional and the Razumikhin-type techniques.

For the successful application of neural networks, it is often a prerequisite to know the actual information about the neuron states which can then be used for optimization or control purposes. However, due to resource and physical limits, it is quite common that only partial information about the neuron states is available through the network outputs especially in relatively large-scale neural networks. As such, an imperative task is to estimate the neural states as precisely as possible via the available network outputs [25], and the resulting state estimation problem for neural networks has attracted an ever-increasing research interest in the past decade and a rich body of literature has been published, see e.g. [3], [5], [7], [20] and the references therein. In particular, a state estimator has been designed in [27] for discrete-time neural networks with Markovian jumping parameters and time-varying delays. The problem of observerbased state estimation has been studied in [15] for fuzzy neural networks (FNNs) with time-varying structured uncertainties and time-varying delays.

In the course of controller/estimator implementation, it is often the case that the actually implemented parameters are slightly different from the expected ones owing to various reasons such as numerical roundoff errors, limited word length of the computer and the imprecision in analogue-digital conversion. It is now a well-known fact that small even tiny variations/drifts of the estimator/filter/controller parameters could lead to dramatic changes (e.g. performance degradation or even eventual instability) of the overall system dynamics [2], [10], and such kind of phenomenon is referred to as the fragility. In the past few years, the non-fragility has become an increasingly important performance index that aims to guarantee that the desired system behavior is insensitive to the admissible implementation errors for the controllers/estimators. For example, in [37], a non-fragile $H_{\infty}$ filter has been designed for a class of discrete-time T-S fuzzy systems with both randomly occurring gain variations (ROGVs) and channel fadings. In [28], the issue of the non-fragile robust finite-time $H_{\infty}$ control has been dealt with for a class of uncertain nonlinear stochastic Itô systems via neural network. In [4], a non-fragile procedure has been introduced to study the problem of synchronization of neural networks with time-varying delay.

In the context of non-fragile estimation for neural networks, some initial results have been appeared in the literature. For example, in [23], the non-fragile observer design problem has been dealt with for neural networks with mixed time-varying delays and Markovian jumping parameters by developing a reciprocal convex approach. Furthermore, the non-fragile state estimation problem has been investigated in [16] for a class of memristive neural networks with two different types of memductance functions and uncertain time-varying delays by using the Wirtinger-type inequality analysis. It should be pointed out that, in [16], [23], the structure of the estimator/observer to be designed has been assumed to be similar to that of the underlying neural network so as to facilitate the subsequent dynamics analysis. Such an 
assumption, however, implies that the time-varying delays and the activation functions would have to be exactly known in order to make practical sense of the estimator/observer implementation. Unfortunately, utilization of such complex estimators/observers is pretty inconvenient as this would place great demands on the parameter identification as well as the engineering realization. As such, a seemingly natural idea is to develop an easy-to-implement state estimator which is of a simple structure so as to facilitate the practical application with guaranteed estimation performance, and this constitutes the main motivation of this paper.

In this paper, we deal with the non-fragile state estimation problem for a class of continuous neural networks with time-varying delays. By employing a combination of the Lyapunov functionals and the matrix analysis techniques, sufficient conditions are established to ensure the existence of the desired estimators and the explicit characterization of such estimators are then given via solving a linear matrix inequality. A simulation example is used to illustrate the effectiveness of the proposed design method. Comparing to the existing results, the novelty of this paper is mainly twofold: 1) the structure of the non-fragile estimator is of a simple linear form that contains two constant gain matrices, thereby facilitating the practical implementation; and 2) the estimator gains are allowed to tolerate multiplicative parameter variations within a given range and such multiplicative form can better reflect the gain-dependent perturbations.

Notation The notation used in this paper is fairly standard except where otherwise stated. $M^{T}$ represents the transpose of $M . \mathbb{R}^{n}$ represents the $n$ dimensional Euclidean space and $\mathbb{R}^{n \times m}$ is the set of all $n \times m$ real matrices. The notation $P>0$ means that $P$ is a real, symmetric, positive definite matrix. $|x|$ stands for the Euclidean norm of a vector $x$. The notation $\operatorname{diag}\left\{A_{1}, A_{2}, \ldots, A_{n}\right\}$ stands for a block-diagonal matrix, and $*$ always denotes the symmetric block in a symmetric matrix. The notation $\lambda_{\max }(\cdot)$ shows the maximum eigenvalue.

\section{PRoblem FORMUlation}

Consider a class of delayed neural network described by:

$$
\dot{x}(t)=-C x(t)+A g(x(t))+B g(x(t-h(t)))
$$

where $x(\cdot)=\left[x_{1}(\cdot), x_{2}(\cdot), \cdots, x_{n}(\cdot)\right]^{T} \in R^{n}$ is the neural state vector, $g(x(\cdot))=\left[g_{1}\left(x_{1}(\cdot)\right), g_{2}\left(x_{2}(\cdot)\right)\right.$, $\left.\cdots, g_{n}\left(x_{n}(\cdot)\right)\right]^{T} \in R^{n}$ is the nonlinear activation function with the initial condition $g(0)=0, C=$ $\operatorname{diag}\left\{c_{1}, c_{2}, \ldots, c_{n}\right\}$ is the positive definite diagonal matrix, $A$ and $B$ are the connection weight matrix and the delayed connection weight matrix, respectively. $h(t)$ is the time-varying delay satisfying

$$
\begin{gathered}
0 \leq h(t) \leq h \\
\dot{h}(t) \leq \mu
\end{gathered}
$$

where $h$ and $\mu$ are constants.

The activation function $g(\cdot)$ satisfies the following Lipschitz condition:

$$
|g(x)-g(y)| \leq|G(x-y)|
$$

where $G=\operatorname{diag}\left\{g_{1}, g_{2}, \ldots, g_{n}\right\}$ is a known diagonal matrix. 
The measurements of the neural network are expressed as follows:

$$
y(t)=D x(t)+f(t, x(t))
$$

where $y(t) \in R^{m}$ is the measurement output, $D \in R^{m \times n}$ is a known constant matrix. $f(t, x(t))$ is a neuronstate-dependent nonlinear perturbation at the output of the network with the initial condition $f(0,0)=0$. $f(t, x(t))$ satisfies the following Lipschitz condition:

$$
\left|f\left(t, x_{1}\right)-f\left(t, x_{2}\right)\right| \leq\left|F\left(x_{1}-x_{2}\right)\right|
$$

where $F=\operatorname{diag}\left\{f_{1}, f_{2}, \ldots, f_{n}\right\}$ is a known constant matrix.

The full-order non-fragile state estimator is of the following form:

$$
\dot{\hat{x}}(t)=\left(A_{F}+\Delta A_{F}\right) \hat{x}(t)+\left(B_{F}+\Delta B_{F}\right) y(t)
$$

where $\hat{x}(t)$ is the state estimation of the neural network, $A_{F}$ and $B_{F}$ are the gain matrices of the estimator to be designed. $\Delta A_{F}$ and $\Delta B_{F}$ quantify the estimator gain variations satisfying the following norm-bounded multiplicative form [11]:

$$
\begin{aligned}
& \Delta A_{F}=A_{F} H_{A} F_{A}(t) E_{A} \\
& \Delta B_{F}=B_{F} H_{B} F_{B}(t) E_{B}
\end{aligned}
$$

where $H_{A}, H_{B}, E_{A}$ and $E_{B}$ are known matrices with appropriate dimensions, $F_{A}(t)$ and $F_{B}(t)$ are unknown matrices satisfying $F_{A}^{T}(t) F_{A}(t) \leq I$ and $F_{B}^{T}(t) F_{B}(t) \leq I$.

Remark 1: As discussed in the introduction, the non-fragile state estimation problem for time-delayed neural networks has stirred some initial research attention. In the existing literature (e.g. [16], [23]), two typical assumptions are that the estimator gain variation is additive and the time-delays are exactly known due to their involvement in the estimator structure. These assumptions are, unfortunately, a bit restrictive in practice. On one hand, a high gain tends to result in a big gain variation during the implementation, which means that the range of the gain variations is largely dependent on the gain itself. As such, the gain variation is usually multiplicative (rather than additive) with respect to the gains. On the other hand, in the case that the exact identification of the time-varying delays is difficult, it is often desirable to have a delay-independent estimator capable of tolerating the delay effects. Therefore, in this paper, a novel delay-independent estimator is introduced in (7)-(9) where the multiplicative gain variations are introduced.

For analysis convenience, we denote

$$
\eta(t)=\left[\begin{array}{ll}
x^{T}(t) & \hat{x}^{T}(t)
\end{array}\right]^{T}, H_{1}(\eta(t))=\left[\begin{array}{ll}
g^{T}(x(t)) & g^{T}(\hat{x}(t))
\end{array}\right]^{T}, H_{2}(t, \eta(t))=\left[\begin{array}{ll}
f^{T}(t, x(t)) & f^{T}(t, \hat{x}(t))
\end{array}\right]^{T} .
$$

Combining estimator (7) with system (1)-(5), we obtain an augmented system as follows:

$$
\dot{\eta}(t)=\mathcal{A} \eta(t)+\mathcal{B} \eta(t)+\mathcal{C} H_{2}(t, \eta(t))+\mathcal{D} H_{1}(\eta(t-h(t)))+\mathcal{E} H_{1}(\eta(t))
$$

where

$$
\begin{aligned}
& \mathcal{A}=\operatorname{diag}\left\{-C, A_{F}+\Delta A_{F}\right\}, \mathcal{D}=\operatorname{diag}\{B, 0\}, \mathcal{E}=\operatorname{diag}\{A, 0\} \\
& \mathcal{B}=\left[\begin{array}{cc}
0 & 0 \\
\left(B_{F}+\Delta B_{F}\right) D & 0
\end{array}\right], \mathcal{C}=\left[\begin{array}{cc}
0 & 0 \\
B_{F}+\Delta B_{F} & 0
\end{array}\right] .
\end{aligned}
$$


From (4) and (6), we have

$$
\begin{gathered}
H_{1}^{T}(\eta(t)) H_{1}(\eta(t)) \leq \eta^{T}(t) \bar{G}^{T} \bar{G} \eta(t) \\
H_{2}^{T}(t, \eta(t)) H_{2}(t, \eta(t)) \leq \eta^{T}(t) \bar{F}^{T} \bar{F} \eta(t)
\end{gathered}
$$

where $\bar{G}=\operatorname{diag}\{G, G\}, \bar{F}=\operatorname{diag}\{F, F\}$.

Definition 1: The augmented system (10) is said to be asymptotically stable if the following is true:

$$
\lim _{t \rightarrow \infty}|\eta(t)|^{2}=0
$$

The objective of this paper is to design a non-fragile state estimator for the continuous neural network (1) to ensure that the augmented system (10) is asymptotically stable.

\section{Main Results}

In this section, we aim to investigate both the stability analysis and the estimator design problem for neural network system (1) with measurement output (5). Before stating our main results, we introduce the following lemma.

Lemma 1: (S-procedure) [1] Let $M=M^{T}, U$ and $V$ be real matrices of appropriate dimensions with $V$ satisfying $V^{T} V \leq I$, then

$$
M+U V W+W^{T} V^{T} U^{T}<0
$$

if and only if there exists a positive scalar $\epsilon$ such that

$$
M+\epsilon U U^{T}+\epsilon^{-1} W^{T} W<0
$$

or, equivalently,

$$
\Pi=\left[\begin{array}{ccc}
M & \epsilon U & W^{T} \\
\epsilon U^{T} & -\epsilon I & 0 \\
W & 0 & -\epsilon I
\end{array}\right]<0 .
$$

The following theorem is given to ensure that the system (10) is asymptotically stable.

Theorem 1: Consider the continuous neural network (1)-(5) subject to time-varying delays, nonlinear perturbations and gain variations. Let the estimator parameters $A_{F}$ and $B_{F}$ be given. The augmented system (10) is asymptotically stable if there exist positive definite matrices $P>0, Q=\left[\begin{array}{ll}Q_{11} & Q_{12} \\ Q_{12}^{T} & Q_{22}\end{array}\right]>0$, $Z>0, S>0$, matrices $N=\left[\begin{array}{ll}N_{1}^{T} & N_{2}^{T}\end{array}\right]^{T}, M=\left[\begin{array}{ll}M_{1}^{T} & M_{2}^{T}\end{array}\right]^{T}, X=\left[\begin{array}{cc}X_{11} & X_{12} \\ X_{12}^{T} & X_{22}\end{array}\right]$ and positive scalars $\varepsilon_{i}(i=1,2,3)$ satisfying the following linear matrix inequality (LMI):

$$
\hat{\Phi}=\left[\begin{array}{ccccccc}
\Phi_{11} & \Phi_{12} & -M_{1} & P \mathcal{E}+Q_{12} & P \mathcal{D} & P \mathcal{C} & h(\mathcal{A}+\mathcal{B})^{T} P \\
* & \Phi_{22} & -M_{2} & 0 & -(1-\mu) Q_{12} & 0 & 0 \\
* & * & -S & 0 & 0 & 0 & 0 \\
* & * & * & Q_{22}-\varepsilon_{1} I & 0 & 0 & h \mathcal{E}^{T} P \\
* & * & * & * & -(1-\mu) Q_{22}-\varepsilon_{2} I & 0 & h \mathcal{D}^{T} P \\
* & * & * & * & * & -\varepsilon_{3} I & h \mathcal{C}^{T} P \\
* & * & * & * & * & * & -h(2 P-Z)
\end{array}\right]<0
$$




$$
\begin{aligned}
& \Psi_{1}=\left[\begin{array}{ll}
X & N \\
* & Z
\end{array}\right] \geq 0 \\
& \Psi_{2}=\left[\begin{array}{cc}
X & M \\
* & Z
\end{array}\right] \geq 0
\end{aligned}
$$

where

$$
\begin{aligned}
& \Phi_{11}=P(\mathcal{A}+\mathcal{B})+(\mathcal{A}+\mathcal{B})^{T} P+Q_{11}+S+\varepsilon_{1} \bar{G}^{T} \bar{G}+\varepsilon_{3} \bar{F}^{T} \bar{F}+h X_{11}+N_{1}+N_{1}^{T}, \\
& \Phi_{12}=h X_{12}-N_{1}+N_{2}^{T}+M_{1}, \\
& \Phi_{22}=-(1-\mu) Q_{11}+\varepsilon_{2} \bar{G}^{T} \bar{G}+h X_{22}-N_{2}-N_{2}^{T}+M_{2}+M_{2}^{T} .
\end{aligned}
$$

Proof: Construct a Lyapunov functional as follows:

$$
V(\eta(t))=V_{1}(\eta(t))+V_{2}(\eta(t))+V_{3}(\eta(t))+V_{4}(\eta(t))
$$

where

$$
\begin{aligned}
& V_{1}(\eta(t))=\eta^{T}(t) P \eta(t), \\
& V_{2}(\eta(t))=\int_{t-h(t)}^{t}\left[\begin{array}{c}
\eta(s) \\
H_{1}(\eta(s))
\end{array}\right]^{T}\left[\begin{array}{ll}
Q_{11} & Q_{12} \\
Q_{12}^{T} & Q_{22}
\end{array}\right]\left[\begin{array}{c}
\eta(s) \\
H_{1}(\eta(s))
\end{array}\right] d s, \\
& V_{3}(\eta(t))=\int_{-h}^{0} \int_{t+\theta}^{t} \dot{\eta}^{T}(s) Z \dot{\eta}(s) d s d \theta, \\
& V_{4}(\eta(t))=\int_{t-h}^{t} \eta^{T}(s) S \eta(s) d s .
\end{aligned}
$$

Calculating the derivatives of $V_{i}(\eta(t))(i=1,2,3,4)$ in (20) along the trajectory of the system (10), we have

$$
\begin{aligned}
\dot{V}_{1}(\eta(t))= & 2 \eta^{T}(t) P \dot{\eta}(t)=2 \eta^{T}(t) P\left[\mathcal{A} \eta(t)+\mathcal{B} \eta(t)+\mathcal{C} H_{2}(t, \eta(t))+\mathcal{D} H_{1}(\eta(t-h(t)))+\mathcal{E} H_{1}(\eta(t))\right], \\
\dot{V}_{2}(\eta(t))= & {\left[\begin{array}{c}
\eta(t) \\
H_{1}(\eta(t))
\end{array}\right]^{T}\left[\begin{array}{ll}
Q_{11} & Q_{12} \\
Q_{12}^{T} & Q_{22}
\end{array}\right]\left[\begin{array}{c}
\eta(t) \\
H_{1}(\eta(t))
\end{array}\right] } \\
& -(1-\dot{h}(t))\left[\begin{array}{c}
\eta(t-h(t)) \\
H_{1}(\eta(t-h(t)))
\end{array}\right]^{T}\left[\begin{array}{ll}
Q_{11} & Q_{12} \\
Q_{12}^{T} & Q_{22}
\end{array}\right]\left[\begin{array}{c}
\eta(t-h(t)) \\
H_{1}(\eta(t-h(t)))
\end{array}\right] \\
\leq & {\left[\begin{array}{c}
\eta(t) \\
H_{1}(\eta(t))
\end{array}\right]^{T}\left[\begin{array}{ll}
Q_{11} & Q_{12} \\
Q_{12}^{T} & Q_{22}
\end{array}\right]\left[\begin{array}{c}
\eta(t) \\
H_{1}(\eta(t))
\end{array}\right] } \\
& -(1-\mu)\left[\begin{array}{c}
\eta(t-h(t)) \\
H_{1}(\eta(t-h(t)))
\end{array}\right]^{T}\left[\begin{array}{cc}
Q_{11} & Q_{12} \\
Q_{12}^{T} & Q_{22}
\end{array}\right]\left[\begin{array}{c}
\eta(t-h(t)) \\
H_{1}(\eta(t-h(t)))
\end{array}\right], \\
\dot{V}_{3}(\eta(t))= & h \dot{\eta}^{T}(t) Z \dot{\eta}(t)-\int_{t-h}^{t} \dot{\eta}^{T}(s) Z \dot{\eta}(s) d s
\end{aligned}
$$




$$
\begin{aligned}
& =h \dot{\eta}^{T}(t) Z \dot{\eta}(t)-\int_{t-h(t)}^{t} \dot{\eta}^{T}(s) Z \dot{\eta}(s) d s-\int_{t-h}^{t-h(t)} \dot{\eta}^{T}(s) Z \dot{\eta}(s) d s, \\
\dot{V}_{4}(\eta(t)) & =\eta^{T}(t) S \eta(t)-\eta^{T}(t-h) S \eta(t-h) .
\end{aligned}
$$

By using the Leibniz-Newton formula, for matrices $M$ and $N$ with appropriate dimensions, we obtain the following equations:

$$
\begin{aligned}
& 0=2 \zeta^{T}(t) N\left[\eta(t)-\eta(t-h(t))-\int_{t-h(t)}^{t} \dot{\eta}(s) d s\right], \\
& 0=2 \zeta^{T}(t) M\left[\eta(t-h(t))-\eta(t-h)-\int_{t-h}^{t-h(t)} \dot{\eta}(s) d s\right]
\end{aligned}
$$

where $\zeta(t)=\left[\eta^{T}(t) \quad \eta^{T}(t-h(t))\right]^{T}$.

On the other hand, according to the conditions (11) and (12), for positive scalars $\varepsilon_{i}(i=1,2,3)$, it can be deduced that

$$
\begin{aligned}
& 0 \leq \varepsilon_{1}\left[\eta^{T}(t) \bar{G}^{T} \bar{G} \eta(t)-H_{1}^{T}(\eta(t)) H_{1}(\eta(t))\right], \\
& 0 \leq \varepsilon_{2}\left[\eta^{T}(t-h(t)) \bar{G}^{T} \bar{G} \eta(t-h(t))-H_{1}^{T}(\eta(t-h(t))) H_{1}(\eta(t-h(t)))\right], \\
& 0 \leq \varepsilon_{3}\left[\eta^{T}(t) \bar{F}^{T} \bar{F} \eta(t)-H_{2}^{T}(t, \eta(t)) H_{2}(t, \eta(t))\right] .
\end{aligned}
$$

For matrix $X=X^{T}$ of any suitable dimension, it is derived that

$$
\begin{aligned}
0 & =\int_{t-h}^{t} \zeta^{T}(t) X \zeta(t) d s-\int_{t-h}^{t} \zeta^{T}(t) X \zeta(t) d s \\
& =h \zeta^{T}(t) X \zeta(t)-\int_{t-h(t)}^{t} \zeta^{T}(t) X \zeta(t) d s-\int_{t-h}^{t-h(t)} \zeta^{T}(t) X \zeta(t) d s .
\end{aligned}
$$

Adding the right-hand sides of the formulas (21)-(26) to the derivatives of $V_{i}(\eta(t))(i=1,2,3,4)$, one obtains that

$$
\dot{V}(\eta(t)) \leq \psi^{T}(t)\left[\Phi_{1}+h \Phi_{2}^{T} Z \Phi_{2}\right] \psi(t)-\int_{t-h(t)}^{t} \xi^{T}(t, s) \Psi_{1} \xi(t, s) d s-\int_{t-h}^{t-h(t)} \xi^{T}(t, s) \Psi_{2} \xi(t, s) d s
$$


where

$$
\begin{aligned}
& \Phi_{1}=\left[\begin{array}{cccccc}
\Phi_{11} & \Phi_{12} & -M_{1} & P \mathcal{E}+Q_{12} & P \mathcal{D} & P \mathcal{C} \\
* & \Phi_{22} & -M_{2} & 0 & -(1-\mu) Q_{12} & 0 \\
* & * & -S & 0 & 0 & 0 \\
* & * & * & Q_{22}-\varepsilon_{1} I & 0 & 0 \\
* & * & * & * & -(1-\mu) Q_{22}-\varepsilon_{2} I & 0 \\
* & * & * & * & * & -\varepsilon_{3} I
\end{array}\right] \\
& \Phi_{2}=\left[\begin{array}{llllll}
\mathcal{A}+\mathcal{B} & 0 & 0 & \mathcal{E} & \mathcal{D} & \mathcal{C}
\end{array}\right], \xi(t, s)=\left[\begin{array}{ll}
\zeta^{T}(t) & \dot{\eta}^{T}(s)
\end{array}\right]^{T}, \\
& \psi(t)=\left[\begin{array}{llllll}
\eta^{T}(t) & \eta^{T}(t-h(t)) & \eta^{T}(t-h) & H_{1}^{T}(\eta(t)) & H_{1}^{T}(\eta(t-h(t))) & H_{2}^{T}(t, \eta(t))
\end{array}\right]^{T} .
\end{aligned}
$$

Note that if $\Phi_{1}+h \Phi_{2}^{T} Z \Phi_{2}<0, \Psi_{1} \geq 0$ and $\Psi_{2} \geq 0$, then for sufficiently small $\varepsilon>0$, we have $\dot{V}(\eta(t))<-\varepsilon\|\eta(t)\|^{2}$ and then the augmented system (10) would be asymptotically stable by the Lyapunov stability theory. Since $\Psi_{1} \geq 0$ and $\Psi_{2} \geq 0$ are true according to (15) and (16), it remains to prove that $\Phi_{1}+h \Phi_{2}^{T} Z \Phi_{2}<0$. By the Schur Complement Lemma, $\Phi_{1}+h \Phi_{2}^{T} Z \Phi_{2}<0$ holds if and only if

$$
\Phi=\left[\begin{array}{ccccccc}
\Phi_{11} & \Phi_{12} & -M_{1} & P \mathcal{E}+Q_{12} & P \mathcal{D} & P \mathcal{C} & h(\mathcal{A}+\mathcal{B})^{T} Z \\
* & \Phi_{22} & -M_{2} & 0 & -(1-\mu) Q_{12} & 0 & 0 \\
* & * & -S & 0 & 0 & 0 & 0 \\
* & * & * & Q_{22}-\varepsilon_{1} I & 0 & 0 & h \mathcal{E}^{T} Z \\
* & * & * & * & -(1-\mu) Q_{22}-\varepsilon_{2} I & 0 & h \mathcal{D}^{T} Z \\
* & * & * & * & * & -\varepsilon_{3} I & h \mathcal{C}^{T} Z \\
* & * & * & * & * & * & -h Z
\end{array}\right]<0 .
$$

Pre- and post-multiplying the inequality (28) by $\operatorname{diag}\left\{I, I, I, I, I, I, P Z^{-1}\right\}$ and $\operatorname{diag}\{I, I, I, I, I$, $\left.I, Z^{-1} P\right\}$, one obtains

$$
\tilde{\Phi}_{1}=\left[\begin{array}{ccccccc}
\Phi_{11} & \Phi_{12} & -M_{1} & P \mathcal{E}+Q_{12} & P \mathcal{D} & P \mathcal{C} & h(\mathcal{A}+\mathcal{B})^{T} P \\
* & \Phi_{22} & -M_{2} & 0 & -(1-\mu) Q_{12} & 0 & 0 \\
* & * & -S & 0 & 0 & 0 & 0 \\
* & * & * & Q_{22}-\varepsilon_{1} I & 0 & 0 & h \mathcal{E}^{T} P \\
* & * & * & * & -(1-\mu) Q_{22}-\varepsilon_{2} I & 0 & h \mathcal{D}^{T} P \\
* & * & * & * & * & -\varepsilon_{3} I & h \mathcal{C}^{T} P \\
* & * & * & * & * & * & -h P Z^{-1} P
\end{array}\right]<0 .
$$

Noticing that $(P-Z)^{T} Z^{-1}(P-Z)=P Z^{-1} P-2 P+Z \geq 0$, we have $P Z^{-1} P \geq 2 P-Z$. Then, it follows from (14) that (29) (or $\Phi_{1}+h \Phi_{2}^{T} Z \Phi_{2}<0$ ) is indeed true. Let $\alpha_{0}=\lambda_{\max }(\hat{\Phi})$. Then, we have $\alpha_{0}<0$ and it follows that

$$
\dot{V}(\eta(t)) \leq \alpha_{0}|\eta(t)|^{2} .
$$

Integrating both sides from 0 to $T>0$ gives

$$
V(\eta(T))-V(\eta(0)) \leq \alpha_{0} T|\eta(t)|^{2}
$$


which results in

$$
|\eta(t)|^{2} \leq-\frac{1}{\alpha_{0} T} V(\eta(0))
$$

It can now be concluded that $|\eta(t)|^{2}$ is convergent, and therefore

$$
\lim _{t \rightarrow \infty}|\eta(t)|^{2}=0
$$

The proof is now complete.

Having conducted the stability analysis in Theorem 1, we are now in a position to deal with the problem of designing the non-fragile estimators. The solution to the design of non-fragile estimators is obtained by the following theorem.

Theorem 2: Consider the continuous neural network (1) with estimator gain variations (7). The augmented system (10) is asymptotically stable if there exist positive scalars $\varepsilon_{i}>0(i=1,2,3)$ and $\tilde{\varepsilon}>0$, positive definite matrices $P=\operatorname{diag}\left\{P_{1}, P_{2}\right\}>0, Q=\left[\begin{array}{ll}Q_{11} & Q_{12} \\ Q_{12}^{T} & Q_{22}\end{array}\right] \geq 0, Z>0, S>0$, matrices $N=\left[\begin{array}{ll}N_{1}^{T} & N_{2}^{T}\end{array}\right]^{T}, M=\left[\begin{array}{ll}M_{1}^{T} & M_{2}^{T}\end{array}\right]^{T}, X=\left[\begin{array}{cc}X_{11} & X_{12} \\ X_{12}^{T} & X_{22}\end{array}\right], Z_{A}$ and $Z_{B}$ satisfying

$$
\tilde{\Phi}=\left[\begin{array}{ccc}
\Xi & \tilde{\mathcal{M}} & \tilde{\varepsilon} \tilde{\mathcal{N}}^{T} \\
* & -\tilde{\varepsilon} I & 0 \\
* & * & -\tilde{\varepsilon} I
\end{array}\right]<0
$$


as well as (15) and (16), where

$$
\begin{aligned}
& \Xi=\left[\begin{array}{ccccccc}
\hat{\Phi}_{11 \Xi} & \Phi_{12} & -M_{1} & A_{1}+Q_{12} & A_{2} & A_{3} & A_{4} \\
* & \Phi_{22} & -M_{2} & 0 & -(1-\mu) Q_{12} & 0 & 0 \\
* & * & -S & 0 & 0 & 0 & 0 \\
* & * & * & Q_{22}-\varepsilon_{1} I & 0 & 0 & A_{5} \\
* & * & * & * & -(1-\mu) Q_{22}-\varepsilon_{2} I & 0 & A_{6} \\
* & * & * & * & * & -\varepsilon_{3} I & A_{7} \\
* & * & * & * & * & * & -h(2 P-Z)
\end{array}\right], \\
& A_{1}=\operatorname{diag}\left\{P_{1} A, 0\right\}, A_{2}=\operatorname{diag}\left\{P_{1} B, 0\right\}, A_{3}=\left[\begin{array}{cc}
0 & 0 \\
Z_{B} & 0
\end{array}\right], A_{4}=\left[\begin{array}{cc}
-h C^{T} P_{1} & h D^{T} Z_{B}^{T} \\
0 & h Z_{A}^{T}
\end{array}\right] \text {, } \\
& A_{5}=\operatorname{diag}\left\{h A^{T} P_{1}, 0\right\}, A_{6}=\operatorname{diag}\left\{h B^{T} P_{1}, 0\right\}, A_{7}=\left[\begin{array}{cc}
0 & h Z_{B}^{T} \\
0 & 0
\end{array}\right] \text {, } \\
& \hat{\Phi}_{11 \Xi}=\left[\begin{array}{cc}
-P_{1} C & 0 \\
Z_{B} D & Z_{A}
\end{array}\right]+\left[\begin{array}{cc}
-C^{T} P_{1} & D^{T} Z_{B}^{T} \\
0 & Z_{A}^{T}
\end{array}\right]+Q_{11}+S+\varepsilon_{1} \bar{G}^{T} \bar{G}+\varepsilon_{3} \bar{F}^{T} \bar{F}+h X_{11}+N_{1}+N_{1}^{T}, \\
& \tilde{\mathcal{M}}=\left[\begin{array}{lllllll}
\tilde{\mathcal{A}}_{1} & \tilde{\mathcal{A}}_{2} & 0 & 0 & 0 & \tilde{\mathcal{A}}_{3} & \tilde{\mathcal{A}}_{4}
\end{array}\right], \tilde{\mathcal{N}}=\left[\begin{array}{lllllll}
\tilde{\mathcal{A}}_{5} & 0 & 0 & 0 & 0 & \tilde{\mathcal{A}}_{6} & 0
\end{array}\right] \text {, } \\
& \tilde{\mathcal{A}}_{1}=\left[\begin{array}{lllllll}
\tilde{\mathcal{M}}_{1}^{T} & 0 & 0 & 0 & 0 & 0 & 0
\end{array}\right]^{T}, \tilde{\mathcal{A}}_{2}=\left[\begin{array}{lllllll}
0 & 0 & 0 & 0 & 0 & 0 & \tilde{\mathcal{M}}_{3}^{T}
\end{array}\right]^{T} \text {, } \\
& \tilde{\mathcal{A}}_{3}=\left[\begin{array}{lllllll}
\tilde{\mathcal{M}}_{2}^{T} & 0 & 0 & 0 & 0 & 0 & 0
\end{array}\right]^{T}, \tilde{\mathcal{A}}_{4}=\left[\begin{array}{lllllll}
0 & 0 & 0 & 0 & 0 & 0 & \tilde{\mathcal{M}}_{4}^{T}
\end{array}\right]^{T} \text {, } \\
& \tilde{\mathcal{A}}_{5}=\left[\begin{array}{lllllll}
\tilde{\mathcal{N}}_{1}^{T} & \tilde{\mathcal{N}}_{2}^{T} & 0 & 0 & 0 & 0 & 0
\end{array}\right]^{T}, \quad \tilde{\mathcal{A}}_{6}=\left[\begin{array}{lllllll}
0 & 0 & 0 & 0 & 0 & \tilde{\mathcal{N}}_{3}^{T} & \tilde{\mathcal{N}}_{4}^{T}
\end{array}\right]^{T}, \\
& \tilde{\mathcal{M}}_{1}=\left[\begin{array}{cc}
0 & 0 \\
Z_{B} H_{B} & Z_{A} H_{A}
\end{array}\right], \tilde{\mathcal{M}}_{2}=\left[\begin{array}{cc}
0 & 0 \\
Z_{B} H_{B} & 0
\end{array}\right], \tilde{\mathcal{M}}_{3}=\left[\begin{array}{cc}
0 & 0 \\
Z_{B} H_{B} & Z_{A} H_{A}
\end{array}\right] \text {, } \\
& \tilde{\mathcal{M}}_{4}=\left[\begin{array}{cc}
0 & 0 \\
Z_{B} H_{B} & 0
\end{array}\right], \tilde{\mathcal{N}}_{1}=\operatorname{diag}\left\{E_{B} D, E_{A}\right\}, \tilde{\mathcal{N}}_{2}=\operatorname{diag}\left\{E_{B} D h, E_{A} h\right\}, \\
& \tilde{\mathcal{N}}_{3}=\operatorname{diag}\left\{E_{B}, 0\right\}, \tilde{\mathcal{N}}_{4}=\operatorname{diag}\left\{E_{B} h, 0\right\},
\end{aligned}
$$

and $\Phi_{12}$ and $\Phi_{22}$ are defined, respectively, in (18) and (19). In this case, the estimator gains in the form of (7) can be obtained by $A_{F}=P_{2}^{-1} Z_{A}$ and $B_{F}=P_{2}^{-1} Z_{B}$.

Proof: According to Theorem 1, to prove the asymptotic stability of the system (10), it suffices to show that (14) is implied by (34). For this purpose, we substitute $\mathcal{A}, \mathcal{B}, \mathcal{C}, \mathcal{D}$ and $\mathcal{E}$ defined in (10) to $\hat{\Phi}$ defined in (14) and obtain

$$
\hat{\Phi}=\left[\begin{array}{ccccccc}
\hat{\Phi}_{11} & \Phi_{12} & -M_{1} & A_{1}+Q_{12} & A_{2} & \bar{A}_{1} & \bar{A}_{2} \\
* & \Phi_{22} & -M_{2} & 0 & -(1-\mu) Q_{12} & 0 & 0 \\
* & * & -S & 0 & 0 & 0 & 0 \\
* & * & * & Q_{22}-\varepsilon_{1} I & 0 & 0 & A_{5} \\
* & * & * & * & -(1-\mu) Q_{22}-\varepsilon_{2} I & 0 & A_{6} \\
* & * & * & * & * & -\varepsilon_{3} I & \bar{A}_{3} \\
* & * & * & * & * & * & -h(2 P-Z)
\end{array}\right]
$$


where

$$
\begin{aligned}
& \bar{A}_{1}=\left[\begin{array}{cc}
0 & 0 \\
P_{2}\left(B_{F}+\Delta B_{F}\right) & 0
\end{array}\right], \bar{A}_{2}=\left[\begin{array}{cc}
-h C^{T} P_{2} & h D^{T}\left(B_{F}+\Delta B_{F}\right)^{T} P_{2} \\
0 & h\left(A_{F}+\Delta A_{F}\right)^{T} P_{2}
\end{array}\right], \\
& \bar{A}_{3}=\left[\begin{array}{cc}
0 & h\left(B_{F}+\Delta B_{F}\right)^{T} P_{2} \\
0 & 0
\end{array}\right] .
\end{aligned}
$$

Furthermore, note that $\hat{\Phi}$ can be decomposed as follows:

$$
\hat{\Phi}=\Xi+\Delta \Xi
$$

where

$$
\begin{aligned}
& \Xi=\left[\begin{array}{ccccccc}
\hat{\Phi}_{11 \Xi} & \Phi_{12} & -M_{1} & A_{1}+Q_{12} & A_{2} & A_{3} & A_{4} \\
* & \Phi_{22} & -M_{2} & 0 & -(1-\mu) Q_{12} & 0 & 0 \\
* & * & -S & 0 & 0 & 0 & 0 \\
* & * & * & Q_{22}-\varepsilon_{1} I & 0 & 0 & A_{5} \\
* & * & * & * & -(1-\mu) Q_{22}-\varepsilon_{2} I & 0 & A_{6} \\
* & * & * & * & * & -\varepsilon_{3} I & A_{7} \\
* & * & * & * & * & * & -h(2 P-Z)
\end{array}\right], \\
& \Delta \Xi=\left[\begin{array}{ccccccc}
\hat{\Phi}_{11 \Delta \Xi} & 0 & 0 & 0 & 0 & A_{8} & A_{9} \\
* & 0 & 0 & 0 & 0 & 0 & 0 \\
* & * & 0 & 0 & 0 & 0 & 0 \\
* & * & * & 0 & 0 & 0 & 0 \\
* & * & * & * & 0 & 0 & 0 \\
* & * & * & * & * & 0 & A_{10} \\
* & * & * & * & * & * & 0
\end{array}\right], \hat{\Phi}_{11 \Delta \Xi}=A_{11}+A_{12} \\
& A_{8}=\left[\begin{array}{cc}
0 & 0 \\
P_{2} \Delta B_{F} & 0
\end{array}\right], A_{9}=\left[\begin{array}{cc}
0 & h D^{T} \Delta B_{F}^{T} P_{2} \\
0 & h \Delta A_{F}^{T} P_{2}
\end{array}\right], A_{10}=\left[\begin{array}{cc}
0 & h \Delta B_{F}^{T} P_{2} \\
0 & 0
\end{array}\right] \text {, } \\
& A_{11}=\left[\begin{array}{cc}
0 & 0 \\
P_{2} \Delta B_{F} D & P_{2} \Delta A_{F}
\end{array}\right], A_{12}=\left[\begin{array}{cc}
0 & D^{T} \Delta B_{F}^{T} P_{2} \\
0 & \Delta A_{F}^{T} P_{2}
\end{array}\right] \text {. }
\end{aligned}
$$

On the other hand, let $Z_{A}=P_{2} A_{F}$ and $Z_{B}=P_{2} B_{F}$. Moreover, $\Delta \Xi$ can be further split as

$$
\begin{aligned}
\Delta \Xi & =\Delta \Xi_{1}+\Delta \Xi_{2} \\
& =\tilde{\mathcal{M}} F_{m} \tilde{\mathcal{N}}+\tilde{\mathcal{N}}^{T} F_{m}^{T} \tilde{\mathcal{M}}^{T}
\end{aligned}
$$

where

$$
\begin{aligned}
& \Delta \Xi_{1}=\left[\begin{array}{lllllll}
\tilde{\mathcal{A}}_{7} & 0 & 0 & 0 & 0 & \tilde{\mathcal{A}}_{8} & 0
\end{array}\right], \Delta \Xi_{2}=\left[\begin{array}{lllllll}
\tilde{\mathcal{A}}_{9} & 0 & 0 & 0 & 0 & 0 & \tilde{\mathcal{A}}_{10}
\end{array}\right], \\
& \tilde{\mathcal{A}}_{7}=\left[\begin{array}{lllllll}
A_{11}^{T} & 0 & 0 & 0 & 0 & 0 & A_{9}
\end{array}\right]^{T}, \tilde{\mathcal{A}}_{8}=\left[\begin{array}{lllllll}
A_{8}^{T} & 0 & 0 & 0 & 0 & 0 & A_{10}
\end{array}\right]^{T} \\
& \tilde{\mathcal{A}}_{9}=\left[\begin{array}{lllllll}
A_{12}^{T} & 0 & 0 & 0 & 0 & A_{8} & 0
\end{array}\right]^{T}, \tilde{\mathcal{A}}_{10}=\left[\begin{array}{lllllll}
A_{9}^{T} & 0 & 0 & 0 & 0 & A_{10}^{T} & 0
\end{array}\right]^{T}, \\
& F_{m}=\operatorname{diag}\left\{F_{m 1}, F_{m 1}, 0,0,0, F_{m 2}, F_{m 2}\right\}, F_{m 1}=\operatorname{diag}\left\{F_{B}(t), F_{A}(t)\right\}, F_{m 2}=\operatorname{diag}\left\{F_{B}(t), 0\right\} .
\end{aligned}
$$


So far, after tedious manipulations, we can rearrange (14) as follows:

$$
\hat{\Phi}=\Xi+\Delta \Xi=\Xi+\tilde{\mathcal{M}} F_{m} \tilde{\mathcal{N}}+\tilde{\mathcal{N}}^{T} F_{m}^{T} \tilde{\mathcal{M}}^{T}<0
$$

It follows from Lemma 1 that (14) is true if there exists a positive scalar $\tilde{\varepsilon}$ such that

$$
\Xi+\tilde{\varepsilon}^{-1} \tilde{\mathcal{M}} \tilde{\mathcal{M}}^{T}+\tilde{\varepsilon}^{-1}(\tilde{\varepsilon} \tilde{\mathcal{N}})^{T}(\tilde{\varepsilon} \tilde{\mathcal{N}})<0
$$

holds. According to the Schur Complement Lemma, we know that (38) holds if and only if (34) is satisfied. To this end, we can conclude that (14) is indeed implied by (34), and it follows from Theorem 1 that the system (10) is asymptotically stable. In addition, the estimator gains are obtained directly from $Z_{A}=P_{2} A_{F}$ and $Z_{B}=P_{2} B_{F}$.

Remark 2: In Theorem 2, sufficient conditions for the asymptotic stability of the dynamic system (10) are presented. It is evident that all the network parameters, the bounds of the activation function, the bounds on the time-delays and its derivatives, the bounds of the nonlinear perturbation and the variations of the gains are reflected in the main results. The corresponding solvability conditions for the desired estimator gains are expressed in terms of the feasibility of a few linear matrix inequalities that can be solved using available software package. It should be pointed out that, compared to existing results in the literature such as [16], [23], the structure of the non-fragile estimator developed in this paper is of a simple linear form containing two constant gain matrices and is therefore convenient for practical implementation. Furthermore, the gain-dependent perturbations, which occur frequently in reality, are taken into account in terms of the multiplicative parameter variations.

\section{A NumericAl EXAMPLE}

In this section, a simulation example is given to verify the effectiveness of the proposed estimator design method for the continuous neural network with time delay and gain variations. Consider a delayed neural network of the form (1) with the parameters as follows:

$C=\left[\begin{array}{ccc}0.2 & 0 & 0 \\ 0 & 0.3 & 0 \\ 0 & 0 & 0.15\end{array}\right], A=\left[\begin{array}{ccc}0.2 & -0.4 & 0.4 \\ -0.4 & 0.2 & 0.2 \\ 0.2 & 0.4 & -0.4\end{array}\right], B=\left[\begin{array}{ccc}0.2 & 0.1 & 0.2 \\ 0.3 & 0.2 & 0.2 \\ 0.2 & 0.2 & 0.1\end{array}\right], h(t)=4, G=0.5 I$

The parameters of the network measurement (5) are given as $D=I, F=0.4 I$, and the parameters of the estimator gain variations are set to be the following

$$
\begin{gathered}
H_{A}=I, H_{B}=I, E_{A}=\left[\begin{array}{lll}
0.3 & 0.3 & 0.3 \\
0.3 & 0.3 & 0.3 \\
0.3 & 0.3 & 0.3
\end{array}\right], E_{B}=\left[\begin{array}{ccc}
0.5 & 0.5 & 0.5 \\
0.5 & 0.5 & 0.5 \\
0.5 & 0.5 & 0.5
\end{array}\right], \\
F_{A}(t)=\operatorname{diag}(\cos (0.1 t), \cos (0.1 t)), F_{B}(t)=\cos (0.1 t) .
\end{gathered}
$$

The selection of the activation functions is as follows:

$$
g_{1}\left(x_{1}(t)\right)=\sin \left(t\left(1-x_{1}(t)\right)\right), g_{2}\left(x_{2}(t)\right)=\sin \left(t\left(1-x_{2}(t)\right)\right), g_{3}\left(x_{3}(t)\right)=\sin \left(t\left(1-x_{3}(t)\right)\right) .
$$

The neuron-state-dependent nonlinear perturbation $f(t, x(t))=0.0004 \cos (x(t))$. 
Using the MATLAB software, the set of solutions to the estimator gain matrices can be obtained as follows:

$$
A_{F}=\left[\begin{array}{ccc}
-0.7359 & 0.2618 & 0.2618 \\
0.2619 & -0.7359 & 0.2618 \\
0.2619 & 0.2618 & -0.7359
\end{array}\right], B_{F}=\left[\begin{array}{ccc}
0.6574 & -0.2743 & -0.2743 \\
-0.2743 & 0.6574 & -0.2743 \\
-0.2743 & -0.2743 & 0.6574
\end{array}\right] .
$$

The simulation results are shown in Figs. 1-4. Figs. 1-3 depict the states of the neural networks $x_{1}(t)$, $x_{2}(t), x_{3}(t)$ and their estimates $\hat{x}_{1}(t), \hat{x}_{2}(t)$ and $\hat{x}_{3}(t)$, respectively. The dynamical evolution of the estimate error is plotted in Fig. 4. The asymptotic stability of the augmented system is confirmed by the simulation results.

\section{CONCLUSIONS}

In this paper, the non-fragile state estimation problem has been investigated for continuous-time neural networks with time-varying delay and nonlinear perturbations. Sufficient conditions that guarantee the asymptotic stability of the augmented system have been obtained by employing the Lyapunov stability theory, and the gain matrices of the estimator have been characterized in terms of the solution to certain LMIs. Finally, the effectiveness of the method has been demonstrated by the simulation results. We would like to point out that our main results can be extended to more general/practical systems such as Itô-type stochastic systems [14], nonlinear Markovian jump systems [18], [22], uncertain stochastic systems [19], sampled-data systems [34] and finite-horizon nonlinear systems [21], [24], and the corresponding results will appear in the near future. In addition, the methods here could be further employed to deal with the fault detection problem [31] as well as the non-fragile state estimation problems for neural networks with more complex phenomena such as fading measurements [29], [30], [32] and incomplete measurements [36], [38].

\section{REFERENCES}

[1] S. Boyd, L. E. Ghaoui, E. Feron and V. Balakrishnan, Linear Matrix Inequalities in System and Control Theory, Philadelphia, PA:SIAM, 1994

[2] P. Dorato, Non-fragile controller design: an overview, Proceedings of American Control Conference, Philadephia, Pennsylvania, pp. 2829-2831, 1998.

[3] V. T. S. Elanayar and Y. C. Shin, Approximation and estimation of nonlinear stochastic dynamic systems using radial basis function neural networks, IEEE Transactions on Neural Networks, Vol. 5, No. 4, pp. 594-603, 1994.

[4] M. Fang and J. H. Park, Non-fragile synchronization of neural networks with time-varying delay and randomly occurring controller gain fluctuation, Applied Mathematics and Computation, Vol. 219, No.15, pp. 8009-8017, 2013.

[5] R. Habtom and L. Litz, Estimation of unmeasured inputs using recurrent neural networks and the extended kalman filter, International Conference on Neural Networks, Houston, Texas, USA, Vol. 4, pp. 2067-2071, 1997.

[6] Y. He, Q. Wang, M. Wu and C. Lin, Delay-dependent state estimation for delayed neural networks, IEEE Transactions on Neural Networks, Vol. 17, No. 4, pp. 1077-1081, 2006.

[7] C. Hua and X. Guan, Output feedback stabilization for time-delay nonlinear interconnected systems using neural networks, IEEE Transactions on Neural Networks, Vol. 19, No. 4, pp. 673-688, 2008.

[8] C. Hua, X. Guan and P. Shi, Robust output feedback tracking control for time-delay nonlinear systems using neural network, IEEE Transactions on Neural Networks, Vol. 18, No. 2, pp. 495-505, 2007.

[9] H. Huang, G. Feng and J. Cao, An LMI approach to delay-dependent state estimation for delayed neural networks, Neurocomputing, Vol. 71, No. 13-15, pp. 2857-2867, 2008.

[10] L. Keel and S. Bhattacharyya, Robust, fragile, or optimal, IEEE Transactions on Automatic Control, Vol. 42, pp. 1098-1105, 1997. 


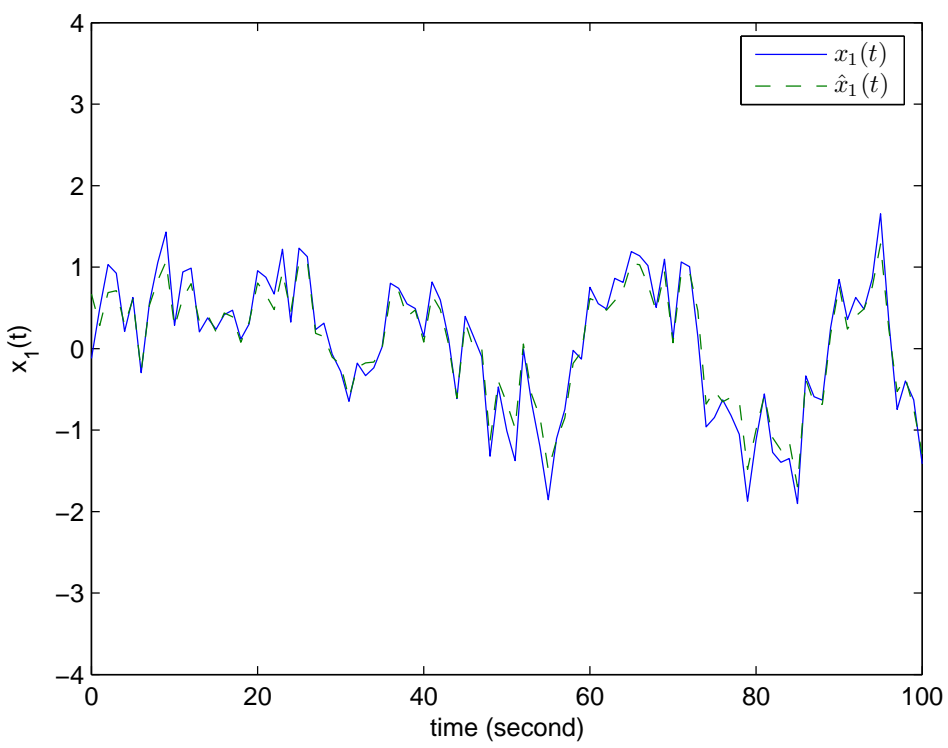

Fig. 1. State vector $x_{1}(t)$ and its estimate $\hat{x}_{1}(t)$.

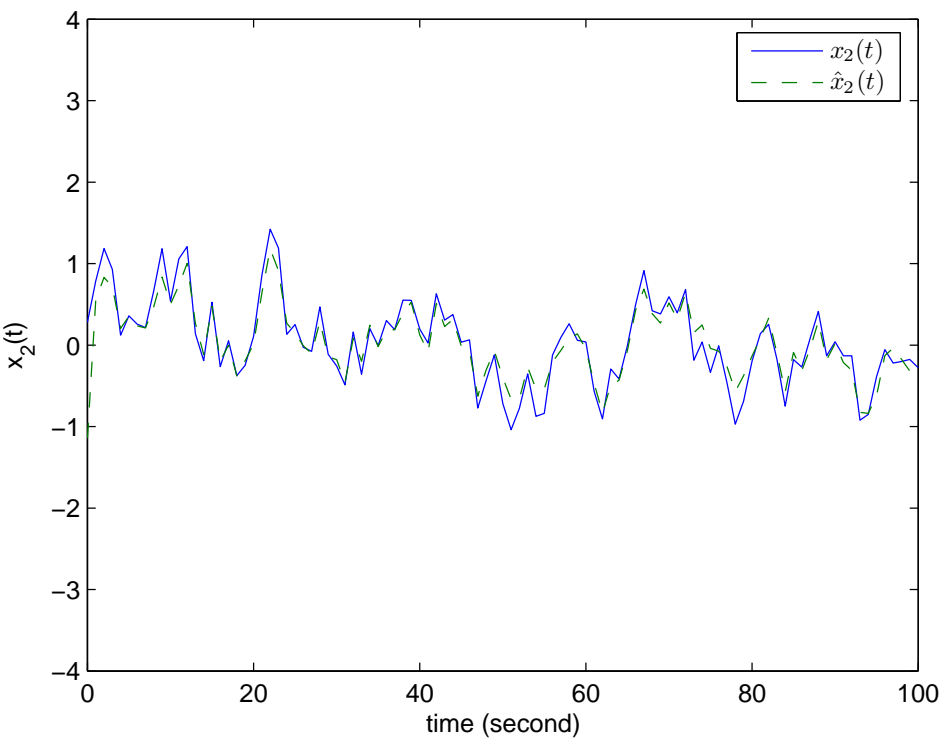

Fig. 2. State vector $x_{2}(t)$ and its estimate $\hat{x}_{2}(t)$.

[11] C. H. Lien, W. C. Cheng, C. H. Tsai and K. W. Yu, Non-fragile observer-based controls of linear system via LMI approach, Chaos, Solitons \& Fractals, Vol. 32, No. 4, pp. 1530-1537, 2007.

[12] J. Jian and Z. Zhao, Global stability in Lagrange sense for BAM-type Cohen-Grossberg neural networks with time-varying delays, Systems Science \& Control Engineering: An Open Access Journal, Vol. 3, No. 1, pp. 1-7, 2015.

[13] Y. Liu, Z. Wang and X. Liu, Global exponential stability of generalized recurrent neural networks with discrete and distributed delays, Neural Networks, Vol. 19, pp. 667-675, 2006.

[14] L. Wu and D. W. C. Ho, Fuzzy filter design for Itô stochastic systems with application to sensor fault detection, IEEE Transactions on Fuzzy Systems, Vol. 17, No. 1, pp. 233-242, 2009.

[15] X. Luo, Q. Ye and B. Cui, Observer-based control for state estimation of uncertain fuzzy neural networks with time-varying delay, Proceedings of the 33rd Chinese Control Conference, Nanjing, China, pp. 5096-5101, 2014.

[16] R. Sakthivel, R. Anbuvithya, K. Mathiyalagan and P. Prakash, Combined $H_{\infty}$ and passivity state estimation of memristive neural 


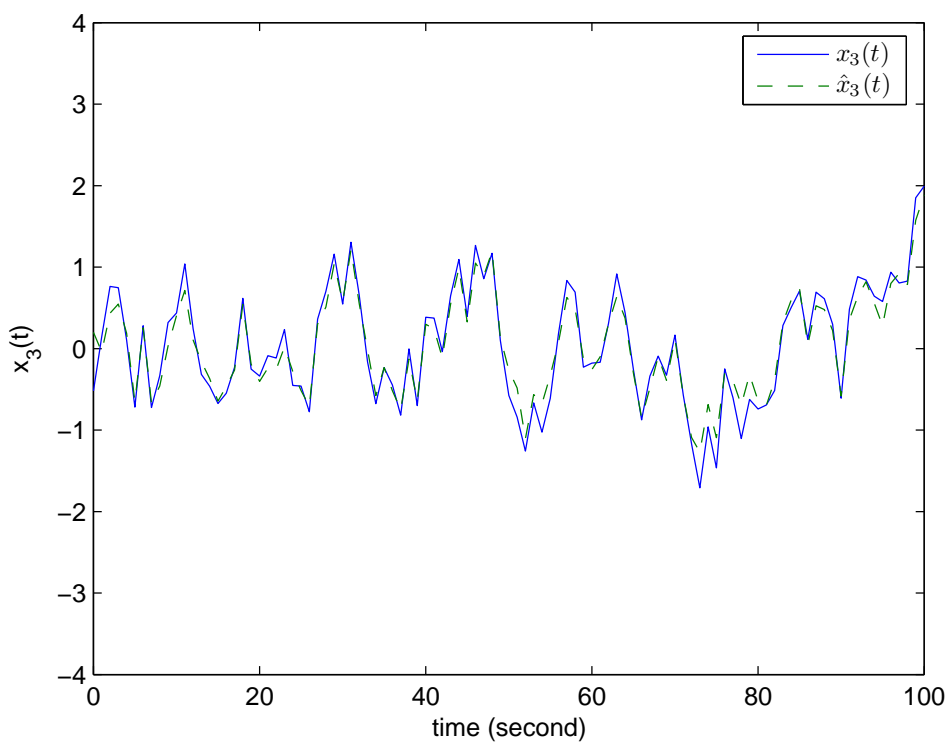

Fig. 3. State vector $x_{3}(t)$ and its estimate $\hat{x}_{3}(t)$.

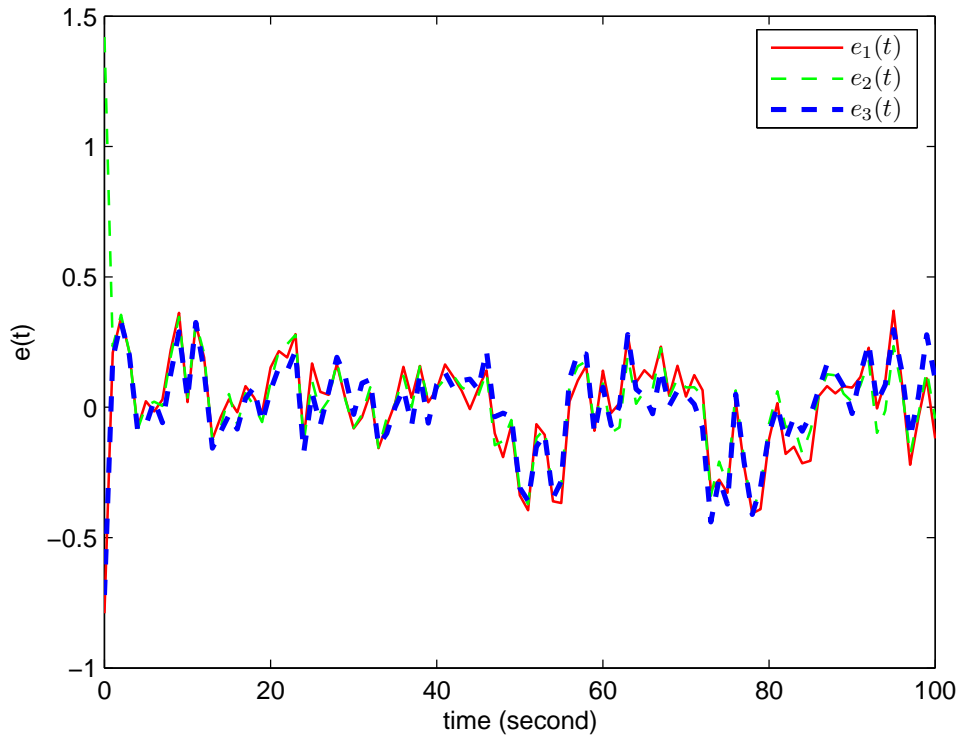

Fig. 4. Error between state vector and its estimate.

networks with random gain fluctuations, Neurocomputing, Vol. 168, pp. 1111-1120, Nov. 2015.

[17] M. Kermania and A. Sakly, Stability analysis for a class of switched nonlinear time-delay systems, Systems Science \& Control Engineering: An Open Access Journal, Vol. 2, No. 1, pp. 80-89, 2014.

[18] L. Ma, Z. Wang, Y. Bo and Z. Guo, Finite-horizon $H_{2} / H_{\infty}$ control for a class of nonlinear Markovian jump systems with probabilistic sensor failures, International Journal of Control, Vol. 84, No. 11, pp. 1847-1857, 2011.

[19] H. Dong, Z. Wang, FE. Alsaadi and B. Ahmad, Event-triggered robust distributed state estimation for sensor networks with statedependent noises, International Journal of General Systems, Vol. 44, No. 2, pp. 254-266, 2015.

[20] F. M. Salam and J. Zhang, Adaptive neural observer with forward co-state propagation, International Joint Conference on Neural Networks, Washington, D.C. Vol. 1, pp. 675-680, 2001.

[21] H. Dong, Z. Wang, SX. Ding and H. Gao, Finite-horizon estimation of randomly occurring faults for a class of nonlinear time-varying systems, Automatica, Vol. 50, No. 12, pp. 3182-3189, 2014. 
[22] L. Wu, X. Yao, and W.X. Zheng, Generalized $H_{2}$ fault detection for Markovian jumping two-dimensional systems, Automatica, Vol. 48, No. 8, pp. 1741-1750, 2012.

[23] V. Vembarasan, P. Balasubramaniam and C. S. Chan, Non-fragile state observer design for neural networks with Markovian jumping parameters and time-delays, Nonlinear Analysis - Hybrid Systems, Vol. 14, pp. 61-73, Nov. 2014.

[24] H. Dong, Z. Wang, SX. Ding and H. Gao, Fnite-horizon reliable control with randomly occurring uncertainties and nonlinearities subject to output quantization, Automatica, Vol. 52, pp. 355-362, 2015.

[25] Z. Wang, D. W. C. Ho and X. Liu, State estimation for delayed neural networks, IEEE Transactions on Neural Networks, Vol. 16, No. 1, pp. 279-284, 2005.

[26] S. L. Wu, K. L. Li and T. Z. Huang, Exponential stability of static neural networks with time delay and impulses, IET Control Theory and Applications, Vol. 5, pp. 943-951, 2011.

[27] Z. Wu, H. Su and J. Chu, State estimation for discrete markovian jumping neural networks with time delay, Neurocomputing, Vol. 73, pp. 2247-2254, 2010.

[28] Z. Yan, G. Zhang and J. Wang, Non-fragile robust finite-time $H_{\infty}$ control for nonlinear stochastic Itô systems using neural network, International Journal of Control, Automation, and Systems, Vol. 10, No. 5, pp. 873-882, 2012.

[29] D. Ding, Z. Wang, B. Shen and H. Dong, $H_{\infty}$ state estimation with fading measurements, randomly varying nonlinearities and probabilistic distributed delays, International Journal of Robust and Nonlinear Control, Vol. 25, No. 13, pp. 2180-2195, 2015.

[30] D. Ding, Z. Wang, J. Lam and B. Shen, Finite-Horizon $H_{\infty}$ control for discrete time-varying systems with randomly occurring nonlinearities and fading measurements, IEEE Transactions on Automatic Control, Vol. 60, No. 9, pp. 2488-2493, 2015.

[31] S. X. Ding, B. Shen, Z. Wang and M. Zhong, A fault detection scheme for linear discrete-time systems with an integrated online performance evaluation, Automatica, Vol. 87, No. 12, pp. 2511-252, 2014.

[32] D. Ding, Z. Wang, B. Shen and H. Dong, Envelope-constrained $H_{\infty}$ filtering with fading measurements and randomly occurring nonlinearities: the finite horizon case, Automatica, Vol. 55, pp. 37-45, 2015.

[33] S. Zhang, H. Liu, D. Gao and W. Wang, Determing the input time delay $\tau$ of a neural network for nonlinear time series prediction, Proceedings of the Second International Conference on Machine Learning and Cybernetics, Xi' an, China, pp. 1255-1258, 2003.

[34] B. Shen, Z. Wang and T. Huang, Stabilization for sampled-data systems under noisy sampling interval, Automatica, Vol. 63, No. 1, pp. 162-166, 2016.

[35] Q. Zhou, P. Shi, S. Xu and H. Li, Observer-based adaptive neural network control for nonlinear stochastic systems with time delay, IEEE Transactions on Neural Networks and learning systems, Vol. 24, No. 1, pp. 71-80, 2012.

[36] B. Shen, Z. Wang, D. Ding and H. Shu, $H_{\infty}$ state estimation for complex networks with uncertain inner coupling and incomplete measurements, IEEE Transactions on Neural Networks and Learning Systems, Vol. 24, No. 12, pp. 2027-2037, 2013.

[37] S. Zhang, Z. Wang, D. Ding, H. Dong, F. Alsaadi and T. Hayat, Non-fragile $H_{\infty}$ fuzzy filtering with randomly occurring gain variations and channel fadings, IEEE Transactions on Fuzzy Systems, DOI: 10.1109/TFUZZ.2015.2446509, 2015.

[38] G. Wei, S. Liu, Y. Song and Y. Liu, Probability-guaranteed set-membership filtering for systems with incomplete measurements, Automatica, Vol. 24, No. 60, pp. 12-16, 2015.

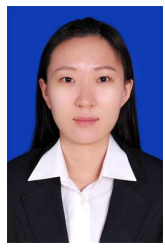

Fan Yang received the B.E. degree in Automation in 2014 from Northeast Petroleum University, Daqing, China, and is currently studying for the M.S. degree in Control Science and Engineering from Northeast Petroleum University. Her research interests include intelligent control systems and neural networks. 


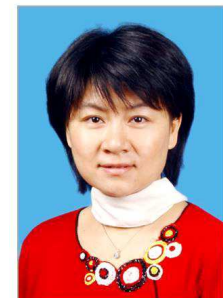

Hongli Dong received the Ph.D. degree in Control Science and Engineering in 2012 from Harbin Institute of Technology, Harbin, China. From July 2009 to January 2010, she was a Research Assistant in the Department of Applied Mathematics, the City University of Hong Kong. From October 2010 to January 2011, she was a Research Assistant in the Department of Mechanical Engineering, the University of Hong Kong. From January 2011 to January 2012, she was a Visiting Scholar in the Department of Information Systems and Computing, Brunel University, London, U.K. From November 2012 to October 2014, she was an Alexander von Humboldt research fellow at the University of Duisburg-Essen, Duisburg, Germany. She is currently a professor with the College of Electrical and Information Engineering, Northeast Petroleum University, Daqing, China.

Dr. Dong's current research interests include robust control and networked control systems. She is a very active reviewer for many international journals.

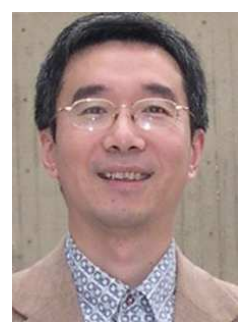

Zidong Wang was born in Jiangsu, China, in 1966. He received the B.Sc. degree in mathematics in 1986 from Suzhou University, Suzhou, China, and the M.Sc. degree in applied mathematics in 1990 and the Ph.D. degree in electrical engineering in 1994, both from Nanjing University of Science and Technology, Nanjing, China.

He is currently Professor of Dynamical Systems and Computing in the Department of Computer Science, Brunel University London, U.K. From 1990 to 2002, he held teaching and research appointments in universities in China, Germany and the UK. Prof. Wang's research interests include dynamical systems, signal processing, bioinformatics, control theory and applications. He has published more than 300 papers in refereed international journals. He is a holder of the Alexander von Humboldt Research Fellowship of Germany, the JSPS Research Fellowship of Japan, William Mong Visiting Research Fellowship of Hong Kong.

Prof. Wang is a Fellow of the IEEE. He is serving or has served as an Associate Editor for 12 international journals, including IEEE Transactions on Automatic Control, IEEE Transactions on Control Systems Technology, IEEE Transactions on Neural Networks, IEEE Transactions on Signal Processing, and IEEE Transactions on Systems, Man, and Cybernetics - Systems. He is also a Fellow of the Royal Statistical Society and a member of program committee for many international conferences.

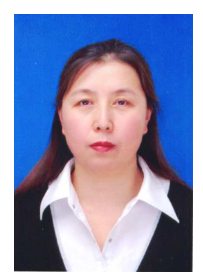

Weijian Ren received the M.S. degree in Control Theory and Control Engineering in 1990 and Ph.D. degree in Oil Storage and Transport Engineering in 2006, both from Northeast Petroleum University, Daqing, China. She is currently a professor at Northeast Petroleum University, Daqing, China. Her research interests include modelling and control of complex systems, fault diagnosis and simulation.

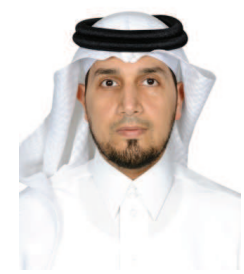

Fuad E. Alsaadi received the B.S. and M.Sc. degrees in electronic and communication from King AbdulAziz University, Jeddah, Saudi Arabia, in 1996 and 2002. He then received the Ph.D. degree in Optical Wireless Communication Systems from the University of Leeds, Leeds, UK, in 2011. Between 1996 and 2005, he worked in Jeddah as a communication instructor in the College of Electronics and Communication. He is currently an assistant professor of the Electrical and Computer Engineering Department within the Faculty of Engineering, King Abdulaziz University, Jeddah, Saudi Arabia. He published widely in the top IEEE communications conferences and journals and has received the Carter award, University of Leeds for the best PhD. He has research interests in optical systems and networks, signal processing, synchronization and systems design. 